\title{
E.-U.GEKELER
}

\section{Sur la géométrie de certaines algèbres de quaternions}

Séminaire de Théorie des Nombres de Bordeaux, tome 2, no 1 (1990), p. $143-153$

<http://www.numdam.org/item?id=JTNB_1990_2_1_143_0>

(C) Université Bordeaux 1, 1990, tous droits réservés.

L'accès aux archives de la revue "Séminaire de Théorie des Nombres de Bordeaux » (http://jtnb.cedram.org/) implique l'accord avec les conditions générales d'utilisation (http://www.numdam.org/conditions). Toute utilisation commerciale ou impression systématique est constitutive d'une infraction pénale. Toute copie ou impression de ce fichier doit contenir la présente mention de copyright.

\section{Numdam}

Article numérisé dans le cadre du programme

Numérisation de documents anciens mathématiques

http://www.numdam.org/ 
Séminaire de Théorie des Nombres, Bordeaux 2 (1990), 143-153

\title{
Sur la géométrie de certaines algèbres de quaternions.
}

\author{
par E.-U. GEKELER
}

1 - Introduction. Soit $K$ un corps global (i.e. un corps de nombres ou un corps de fonctions d'une variable sur un corps fini), et $S$ un ensemble non vide de places de $K$ contenant les places archimédiennes. $A=A_{S}$ désignera toujours l'anneau des $S$-entiers de $K$. Soit $D$ une algèbre de quaternions sur $K$ [9]. Nous appellerons brèvement ordre dans $D$ tout $A$ ordre maximal $B$ dans $D$. Les faits suivants sont bien connus :

(1.1) Le nombre de classes d'idéaux à gauche de $B$ est fini et ne dépend que de $D$ et $S$, mais non du choix de $B ; S$ étant fixé, on l'appelle $h(D)$.

(1.2) Soit $\mathfrak{b}$ un idéal à gauche de $B$ et $B_{d}(\mathfrak{b})=\{f \in D \mid \mathfrak{b} f \subset \mathfrak{b}\}$. Alors, $B_{d}(\mathfrak{b})$ est un ordre (l'ordre à droite de $\mathfrak{b}$ ), i.e. maximal. A conjugaison près, chaque ordre intervient de telle manière.

(1.3) Le nombre $t(D)$ de types de $D$ (=classes de conjugaison d'ordres) est fini et inférieur ou égal à $h(D)$.

Le problème fondamental de l'arithmétique de $D$ est donc de décrire les ensembles finis de classes d'idéaux de $B$ et de types de $D$.

Dans un cas assez spécial (voir sect. 2), on a une belle interprétation de ces ensembles, reliant l'arithmétique dans $D$ à la théorie des courbes elliptiques [4]. Nous donnerons une interprétation similaire de certaines algèbres sur les corps de fonctions, où les modules de Drinfeld remplacent les courbes elliptiques.

Supposons maintenant que $K$ soit un corps global de fonctions sur le corps de constantes $F_{q}$ à $q$ éléments, et que $S$ consiste en une seule place " $\infty$ " de degré $d_{\infty}$ sur $F_{q}$. Soit $\mathfrak{p}$ une place de $K$ différente de $\infty$, de degré $d$, et $D=D(\mathfrak{p})$ l'algèbre de quaternions sur $K$ qui se ramifie en $\mathfrak{p}$ et $\infty$. Ecrivons la fonction zêta de $K$ dans la forme

$$
\zeta_{K}(S)=\frac{P\left(q^{-s}\right)}{\left(1-q^{s}\right)\left(1-q^{1-s}\right)}
$$

avec un polynôme à coefficients entiers. On sait que le cardinal $h=$ \#(Pic $A)$ du groupe de classes Pic $A$ de $A$ est donné par

$$
h=d_{\infty} \cdot P(1) .
$$

Notre interprétation de $D$ impliquera le

Manuscrit reçu le 23 novembre 1989 
THÉORÈme 1. Soit $B$ un ordre dans $D$.

(i) Pour chaque idéal à gauche $\mathfrak{b}$ de $B$, l'ordre $B_{d}(\mathfrak{b})$ a un groupe d'unités isomorphe au groupe multiplicatif de $F_{q}$ ou de son extension quadratique $\mathrm{F}_{q^{2}}$. Soient $h_{1}(D), h_{2}(D)$ les nombres de classes correspondants de $B$ (qui ne dépendent pas du choix de $B$ ).

(ii) Si $d$ ou $d_{\infty}$ est pair, on a

$$
h_{1}(D)=d_{\infty} \cdot P(1) \cdot P(q) \cdot Q \text { et } h_{2}(D)=0 .
$$

Si $d$ et $d_{\infty}$ sont impairs, on a

$$
h_{1}(D)=d_{\infty} \cdot P(1)[P(q) \cdot Q-P(-1) /(q+1)] \text { et } h_{2}(D)=d_{\infty} \cdot P(1) \cdot P(-1) \text {. }
$$

Ici, on a posé

$$
Q=\frac{\left(q^{d}-1\right)\left(q^{d_{\infty}}-1\right)}{(q-1)\left(q^{2}-1\right)}
$$

(iii) Dans chaque cas, on a la formule de masse

$$
\sum w(\mathfrak{b})^{-1}=d_{\infty} \cdot P(1) \cdot P(q) \cdot Q
$$

où la somme porte sur un système de représentants des classes d'idéaux à gauche de $B$, et $w(\mathfrak{b})=\#\left(B_{d}^{*}(\mathfrak{b}): A^{*}\right)$.

(1.6) Remarque. Evidemment, (iii) est une conséquence triviale de (i) et (ii). Le point essentiel c'est que le terme à droite de (iii) a une signification universelle. Il est égal à $h(A) \cdot \zeta_{S^{*}}(-1)$, où $\zeta_{S^{*}}$ est la fonction zêta de $K$, privée des facteurs d'Euler qui correspondent aux places dans $S^{*}=\{\mathfrak{p}, \infty\}$.

\section{Courbes elliptiques supersingulières.}

Pour motiver ce qui suit, nous rappelons la relation (aujourd'hui bien connue) entre algèbres de quaternions sur $\mathbb{Q}$ et courbes elliptiques en caractéristique positive.

Soit $E$ une courbe elliptique (= variété abélienne de dimension un) définie sur la clôture algébrique $\bar{F}_{p}$ du corps fini $F_{p}$, où $p$ est un nombre premier. Nous supposons $E$ suṛersingulière, i.e. elle ne possède aucun point de $p$ torsion non nul. D'ailleurs, soit $D=D(p)$ l'algèbre de quaternions qui se ramifie à $p$ et à la place archimédienne. D'après Deuring et Eichler [4], on sait que

(2.1) L'anneau $B=\operatorname{End}(E)$ des endomorphismes de $E$ est (isomorphe à) un $\mathbb{Z}$-ordre maximal dans $D$ (i.e. un "ordre", si l'on pose $S=\{\infty\}, A=\mathbb{Z}$ ). 
(2.2) Soit $\mathfrak{b}$ un idéal à gauche de $B$ et $H(\mathfrak{b}) \subset E$ le schéma en groupes fini $\cap \operatorname{Ker}(b)$, où $b$ parcourt $\mathfrak{b}$. Le quotient $E(\mathfrak{b})=E / H(\mathfrak{b})$ est une courbe elliptique supersingulière et $\mathfrak{b} \mapsto E(\mathfrak{b})$ définit une bijection entre l'ensemble des classes d'idéaux à gauche de $B$ et l'ensemble $\sum(p)$ des classes d'isomorphisme de courbes elliptiques supersingulières sur $\overline{\mathrm{F}}_{p}$.

(2.3) $B_{d}(\mathfrak{b})$ est isomorphe à $\operatorname{End}(E(\mathfrak{b}))$.

(2.4) $B_{d}(\mathfrak{b})$ et $B_{d}\left(\mathfrak{b}^{\prime}\right)$ sont isomorphes si et seulement si $E(\mathfrak{b}) E\left(\mathfrak{b}^{\prime}\right)$ définissent des classes dans $\sum(p)$ qui sont conjuguées sous le groupe de Galois $\operatorname{Gal}\left(\overline{\mathbf{F}}_{p}: \mathbf{F}_{p}\right)$.

(2.5) Remarque. D'après le théorème de Skolem-Noether, deux ordres isomorphes sont en fait conjugués dans $D$. De plus, les éléments de $\sum(p)$ sont définis sur l'extension quadratique $F_{q}$ de $F_{p}, q=p^{2}$. Donc (2.4) dit que les types de $D$ correspondent aux orbites de $\sum(p)$ sous l'action de $\operatorname{Gal}\left(\mathbf{F}_{q}: \mathbf{F}_{p}\right)$.

Considérons maintenant le schéma modulaire "de Hecke" $M_{0}(p)[3]$. C'est un schéma normal sur $\mathbb{Z}$ qui paramétrise les courbes elliptiques munies d'un sous-schéma en groupes fini de degré $p$. On a $M_{0}(p)(\mathbb{C})=\Gamma_{0}(p) \backslash H$ avec le demi-plan $H$ de Poincaré et le groupe $\Gamma_{0}(p)=\left\{\left(\begin{array}{ll}a & b \\ c & d\end{array}\right) \in S L(2, \mathbb{Z}) \mid c \equiv\right.$ $0(p)$ \}, qui opère sur $H$. La fibre spéciale $M_{0}(p) \times \bar{F}_{p}$ a une jolie description : c'est une courbe avec deux composantes irréductibles, toutes deux isomorphes à la $j$-droite affine $(j=$ l'invariant modulaire des coubes elliptiques), et qui se coupent transversalement aux points supersinguliers. En particulier, le genre arithmétique $p_{a}$ de $\bar{M}_{0}(p) \times \overline{\mathrm{F}}_{p}$ (où $\bar{M}_{0}(p)$ est la compactification naturelle de $\left.M_{0}(p)\right)$ se calcule comme

$$
p_{a}=\#(\text { points doubles })-1=h(D)-1 .
$$

De l'autre côté, $p_{a}$ est égale au genre de la surface de Riemann $\bar{M}_{0}(p)(\mathbb{C})$, qui est facilement calculé à l'aide de la formule de Hurwitz. Il en résulte des formules explicites pour $h(D(p))=\#(\Sigma(p))$ et pour $t(D(p))$, voir e.g. [1]. De plus, on a la formule de masse

$$
\sum w(\mathfrak{b})^{-1}=\left(p-1 / 12=\zeta_{S^{*}}(-1)\right.
$$

où $\mathfrak{b}$ parcourt l'ensemble des classes d'idéaux à gauche de

$$
B=\operatorname{End}(E), w(\mathfrak{b})=\#\left(B_{d}(\mathfrak{b})^{*}: \mathbb{Z}^{*}\right)=\#(\operatorname{Aut}(E(\mathfrak{b})):\{ \pm 1\})
$$


et $S^{*}=\{p, \infty\}$.

\section{Modules de Drinfeld.}

Nous allons donner une interprétation géométrique analogue pour certaines algèbres de quaternions sur les corps de fonctions. A partir de maintenant, nous supposons que $K$ soit un corps global de fonctions avec comme corps de constantes le corps $F_{q}$ ( $q$ puissance d'un nombre premier $p$ ), et que $S$ consiste d'une seule place " $\infty$ " de $K$. Soit $|?|=\mid$ ? $\left.\right|_{\infty}$ la valeur absolue normalisée correspondante, donnée sur un élément non nul de $A$ par $|a|=\#(A / a)$. Dans ce contexte, Drinfeld [5] a introduit la notion des modules elliptiques, que nous appellerons des modules de Drinfeld. Ceux-ci sont des objets diophantiens qui ressemblent beaucoup aux courbes elliptiques. Soit $L$ un corps muni d'une structure $\gamma: A \rightarrow L$ de $A$-algèbre, et $\tau$ l'endomorphisme $x \mapsto x^{q}$ du schéma en groupes additif $G_{a} \mid L$. Rappelons que l'anneau des endomorphismes $\operatorname{End}_{L}\left(G_{a}\right)$ de $G_{a} \mid L$ est l'anneau des polynômes additifs $\sum a_{i} X^{p^{i}}$ à coefficients dans $L$, où la "multiplication" est définie par insertion des polynômes. Soit $L\{\tau\} \subset \operatorname{End}_{L}\left(G_{a}\right)$ la sousalgèbre engendrée par $\tau$. C'est un "anneau de polynômes non-commutatif" avec la règle de commutation $\tau a=a^{q} \tau$ si $a \in L$. Au "polynôme" $\sum a_{i} \tau^{i}$ correspond l'opérateur $x \mapsto \sum a_{i} x^{q^{\prime}}$ sur $G_{a} \mid L$. Un $A$-module de Drinfeld de rang $r$ sur $L$ est une structure de $A$-module sur $G_{a} \mid L$, donnée par un homomorphisme d'anneaux

$$
\begin{aligned}
\Phi: A & \rightarrow L\{\tau\}, \\
a & \mapsto \Phi_{a}=\sum a_{i} \tau^{i}
\end{aligned}
$$

tel que pour chaque $a$ non nul, les deux conditions sont satisfaites :

(3.1) (i) $a_{0}=\gamma(a)$, (ii) le degré du polynôme additif correspondant $\sum a_{i} X^{q^{i}}$ est égal à $|a|^{r}$.

(3.2)Exemple. Si $K=\mathrm{F}_{q}(T)$ et $\infty$ est la place à l'infini usuelle, $A$ est l'anneau $F_{q}[T]$ des polynômes en $T$. Un module de Drinfeld de rang $r$ est donné par $\Phi_{T}=\sum a_{i} \tau^{i}$, où $a_{0}=\gamma(T)$ et $a_{r} \neq 0$.

Un morphisme $u: \Phi \rightarrow \Psi$ de modules de Drinfeld (défini sur $L$ ) est un élément $u$ de $\operatorname{End}_{L}\left(G_{a}\right)$ tel que $u \circ \Phi_{a}=\Psi_{a} \circ u, a \in A$. Donc l'anneau $\operatorname{End}(\Phi)=\operatorname{End}_{L}(\Phi)$ des $L$-endomorphismes de $\Phi$ est le centralisateur de $\Phi(A)$ dans $\operatorname{End}_{L}\left(G_{a}\right)$ (en fait, dans $L\{\tau\}$ ). Pour $a$ non nul, le sous-schéma en $A$-modules ${ }_{a} \Phi=\operatorname{Ker}\left(\Phi_{a}\right)$ de $G_{a} \mid L$ est fini de degré $|a|^{r}$. C'est le schéma des points de a-torsion de $\Phi$. Ecrivons $\operatorname{car}_{A}(L)=\infty$, si $\gamma$ est injectif, et $\operatorname{Ker}(\gamma)$ dans le cas contraire. Si de plus, $a$ est premier à $\operatorname{car}_{A}(L),{ }_{a} \Phi$ 
est réduit, et ses points ${ }_{a} \Phi(\bar{L})$ sur la clôture algébrique de $L$ forment un $A$-module abstrait qui est libre de dimension $r$ sur $A / a$. Tout ce qu'on vient d'expliquer se généralise au schéma $a$ des points de a-torsion de $\Phi$, où a est un idéal non nécessairement principal de $A$. On utilise ces faits à définir des structures de niveau sur les modules de Drinfeld, puis à construire des schémas de modules, ce qui est tout à fait analogue aux constructions similaires dans la théorie des courbes elliptiques. (Voir [5] et [2] pour les détails).

\section{Relation avec les ordres.}

Dans ce qui suit, on écrit "module de Drinfeld", ou "module de D." pour "module de Drinfeld de rang deux". Nous fixons un idéal maximal $\mathfrak{p}$ de $A$, de degré $d$ et d'ordre $m$ dans le groupe de classes Pic A de A. Nous écrivons $\mathrm{F}_{\mathrm{p}}=A / \mathfrak{p}$, et plus généralement $\mathrm{F}_{\mathrm{p}}^{(i)}$ pour l'extension de degré $i$ de $F_{p}$. Un module de $D$. $\Phi$ sur une extension $L$ de $F_{p}$ est dit supersingulier si le schéma des points de $\mathfrak{p}$-torsion $p \Phi$ est local, ou ce qui est équivalent, si $f(\bar{L})=0$ pour un $f$ dont le diviseur est une puissance de $\mathfrak{p}$.

(4.1) Remarque. D'après [8], Cor. 5.2, $\mathrm{F}_{\mathfrak{p}}^{(m)}$ est l'extension minimale de $F_{p}$ sur laquelle existent des modules de $D$. Chaque module supersingulier peut être défini sur $F_{p}^{(2 m)}$ (loc. cit. Prop. 4.2).

Soit $D=D(\mathfrak{p})$ l'algèbre de quaternions sur $K$ qui se ramifie à $\mathfrak{p}$ et $\infty$. Le théorème suivant (comparer aux assertions (2.1) - (2.4)!) est un cas spécial du Thm. 4.3 de [8].

ThÉorème 2. Soit $\Phi$ un module de Drinfeld supersingulier (de rang 2) sur la clôture algébrique $\bar{F}_{p}$ de $F_{p}$.

(i) L'anneau des endomorphismes $B=\operatorname{End}(\Phi)$ est isomorphe à un $A$-ordre maximal dans $D(\mathfrak{p})$.

(ii) Il existe une bijection canonique $(\mathfrak{b}) \mapsto\left(\Phi^{\mathfrak{b}}\right)$ de l'ensemble des classes d'idéaux à gauche de $B$ sur l'ensemble $\sum(\mathfrak{p})$ des classes d'isomorphisme des modules de $D$. supersinguliers sur $\bar{F}_{p}$.

(iii) L'ordre à droite $B_{d}(\mathfrak{b})$ de $\mathfrak{b}$ dans $B \otimes K=D$ est isomorphe à $\operatorname{End}\left(\Phi^{\mathfrak{b}}\right)$.

(iv) Supposons que Pic $A$ soit engendré par la classe de $\mathfrak{p}$. Alors, $B_{d}(\mathfrak{b})$ et $B_{d}\left(\mathfrak{b}^{\prime}\right)$ sont isomorphes si et seulement si $\Phi^{\mathfrak{b}}$ et $\Phi^{\mathfrak{b}^{\prime}}$ définissent des classes dans $\sum(\mathfrak{p})$ qui sont conjuguées sous $\operatorname{Gal}\left(\bar{F}_{\mathrm{p}}: \mathrm{F}_{\mathrm{p}}\right)$. 
Donc il nous reste à décrire l'ensemble $\sum(\mathfrak{p})$ sur un schéma de modules convenable.

\section{Schémas de modules.}

Considérons le schéma grossier de modules "de type Hecke" $M_{0}(\mathfrak{p})$. II paramétrise les classes d'isomorphisme des paires $(\Phi, U)$, où $\Phi$ est un module de $D$. de rang 2 , et $U$ un sous-schéma en $A$-modules de $p_{p} \Phi$ qui est fini de degré $q^{d}$. (Dans la terminologie de [7], I, §3, $M_{0}(\mathfrak{p})$ est le schéma $M_{\mathfrak{K}}^{2}$, où $\mathfrak{K}$ est le sous-groupe de congruence de Hecke $\mathfrak{K}=\mathfrak{K}_{0}(\mathfrak{p})=\left\{\left(\begin{array}{ll}a & b \\ c & d\end{array}\right) \in\right.$ $G L(2, \hat{A}) \mid c \equiv 0(\mathfrak{p})\}$.) D'ailleurs, soit $M(1)$ le schéma grossier pour les modules de rang 2 sans structure de niveau, et $\bar{M}_{0}(\mathfrak{p}), M(1)$ les compactifications canoniques ([5], sect. 9). Ce sont des schémas projectifs sur Spec $A$, obtenus en compactifiant $M_{0}(\mathfrak{p}), M(1)$ fibre par fibre sur Spec $A$. Alors, (5.1) $-\bar{M}_{0}(\mathfrak{p}), \bar{M}(1)$ sont des schémas normaux, irréductibles, de type fini sur Spec A, et $M_{0}(\mathfrak{p}) \subset \bar{M}_{0}(\mathfrak{p}), M(1) \subset \bar{M}(1)$ sont partout denses ;

- le morphisme canonique $\bar{M}_{0}(\mathfrak{p}) \rightarrow \bar{M}(1)$ est plat et fini de degré $q^{d}+1$;

- le morphisme structural $\bar{M}(1) \rightarrow$ Spec $A$ est lisse de dimension un (voir [5], 5.4 et 9.3).

En comparant notre situation avec celle de la section 2, il y a une importante différence, qui résulte de l'existence d'idéaux non-principaux dans $A$. Soit $h=\#($ Pic $A)$ le nombre de classes de $A$, et posons $H$ pour le corps de classes de Hilbert de $(K, \infty)$, c'est-à-dire l'extension maximale abélienne non-ramifiée de $K$ qui se décompose complètement à l'infini. L'application d'Artin identifie Pic $A$ à $\operatorname{Gal}(H: K)$, et l'idéal p se décompose dans l'anneau $A_{H}$ des $A$-entiers de $H$ en $s$ idéaux différents, où $s=h / m$. Contrairement à la section $2, K=\operatorname{Fract}(A)$ n'est pas algébriquement clos dans le corps de fonctions de $\bar{M}(1)$ (resp. $\bar{M}_{0}(\mathfrak{p})$.) Plus précisément, il résulte de [7], II, §4 que :

(5.2) Le normalisé de $\operatorname{Spec} A$ dans ces deux schémas est isomorphe à $\operatorname{Spec}\left(A_{H}\right)$.

Dans le langage traditionnel des variétés, on dirait que "la courbe algébrique $\bar{M}(1)$ est définie sur $H "$, de même pour $\bar{M}_{0}(\mathfrak{p})$.

\section{La fibre spéciale.}

Les arguments de cette section sont essentiellement ceux de [6], eux mêmes inspirés par [3], où on peut trouver plus de détails.

Notons $X(1), \bar{X}(1), X_{0}(\mathfrak{p}), \bar{X}_{0}(\mathfrak{p})$ les produits fibrés sur $A$ avec $\overline{\boldsymbol{F}}_{\mathfrak{p}}$ de 
$M(1), \bar{M}(1), M_{0}(\mathfrak{p}), \bar{M}_{0}(\mathfrak{p}) ; X(1)$ et $X_{0}(\mathfrak{p})$ paramétrisent les modules de Drinfeld $\Phi$, (muni d'un sous- $A$-module $U$ de degré $q^{d} \operatorname{de}_{p} \Phi$ dans le dernier cas) $\operatorname{sur} \overline{\mathbf{F}}_{\mathrm{p}}$.

Soit $F=\tau^{d}$ le morphisme de Frobenius qui correspond à $F_{\mathrm{p}}$. Avoir donné $\Phi$, on a $F: \Phi \rightarrow \Phi^{F}$ où $\Phi^{F}$ est le module $\Phi$ tordu par $F$ (i.e., le module obtenu en appliquant l'élément correspondant de $\operatorname{Gal}\left(\overline{\boldsymbol{F}}_{\mathrm{p}}: \bar{F}_{\mathrm{p}}\right)$ aux coefficients de $\Phi)$. Le noyau $\operatorname{Ker}(F)$ de $F$, qui est strictement local, est contenu dans ${ }_{p} \Phi$, donc il existe une section canonique (en fait, une immersion fermée)

$$
i: X(1) \rightarrow X_{0}(\mathfrak{p})
$$

de la projection

$$
\pi: X_{0}(\mathfrak{p}) \rightarrow X(1),
$$

qui sur les objets des problèmes de modules est donnée par $\Phi \mapsto(\Phi, \operatorname{Ker}(F))$. Cette application est bijective sur les objets supersinguliers, car pour de tels $\Phi$, il n'existe qu'un seul sous- $A$-module

$U$ d'ordre $q^{d}$ dans ${ }_{p} \Phi$. Nous avons encore besoin de l'application

$$
w: X_{0}(\mathfrak{p}) \rightarrow X_{0}(\mathfrak{p})
$$

définie $\operatorname{par}(\Phi, U) \mapsto\left(\Phi / U,{ }_{p} \Phi / U\right)$. Ici, $\Phi / U$ est le module $\Psi$, but d'un morphisme $u: \Phi \rightarrow \Psi$ à noyau $U$, qui est déterminé à isomorphisme près, et $p \Phi / U$ l'image de ${ }_{p} \Phi$ sous $u$. (En général, $w$ n'est pas une involution, mais on a toujours $w^{2 m}=i d$.). Finalement, nous écrivons

$$
F_{X(1)}: X(1) \rightarrow X(1)
$$

pour l'endomorphisme donné par $\Phi \mapsto \Phi^{F}$. On a

$$
\pi \circ i=i d_{X(1)} \text { et } \pi \circ w \circ i=F_{X(1)} .
$$

Si $\Phi$ n'est pas supersingulier, $U$ est soit réduit, soit local, donc $\operatorname{im}(i) \cup \operatorname{im}(w \circ i)=X_{0}(\mathfrak{p})$ et $\operatorname{im}(i) \cap \operatorname{im}(w \circ i)$ consiste en des modules supersinguliers. Cela entraîne (comparer avec [3], V, §1 et VI, §6) :

(6.3) $X_{0}(\mathfrak{p})$ est l'union de deux copies de $X(1)$, qui se coupent transversalement aux points supersinguliers. 
(La transversalité vient du fait évident que $F_{X(1)}$ est purement inséparable.) On recolle le point supersingulier $\Phi$ de la deuxième copie au point $\Phi^{F}$ de la première.

Parce que $\bar{M}(1) \times_{A} \mathrm{~F}_{\mathrm{p}}=\amalg \bar{M}(1) \times_{A_{H}} \mathrm{~F}_{\mathrm{q}_{i}}$ est lisse sur $\mathrm{F}_{\mathrm{p}}, \bar{X}(1)=\bar{M}(1) \times$ $\bar{F}_{p}$ est l'union disjointe de $h=s . m$ courbes algébriques, lisses, connexes et projectives. Vu que les composantes sont permutées par

Pic $A=\operatorname{Gal}(H: K)$, il est évident que chacune contient des points supersinguliers. Le genre commun de ces courbes est

$$
g(1)=\operatorname{genre}\left(\bar{M}(1) \times_{A_{H}} H\right) .
$$

Il existe des extensions uniques $\bar{\pi}, \bar{i}, \bar{w}, F_{\bar{X}(1)}$ aux compactifications $\bar{X}(1), \bar{X}_{0}(\mathfrak{p})$ des morphismes $\pi, i, w, F_{X(1)}$, et les relations (6.2) restent valables: Donc $\bar{X}_{0}(\mathfrak{p})$ est l'union de deux copies de $\bar{X}(1)$. On dérive de la propriété modulaire de $\bar{M}_{0}(\mathfrak{p})([5]$, sect. 9) que $\bar{i}(\bar{X}(1) \backslash X(1))$ et $\bar{w} \circ \bar{i}(\bar{X}(1) \backslash X(1))$ sont disjoints. Cela entraîne que les composantes connexes de $\bar{X}_{0}(\mathfrak{p})$ sont de la forme $X \cup Y$, où $X=F_{\bar{X}(1)}(Y)$ et $Y$ sont des composantes de $\bar{X}(1)$ recollées en les points supersinguliers comme cela est décrit plus haut. Le genre arithmétique se calcule par

$$
p_{a}\left(\bar{X}_{0}(\mathfrak{p})\right)=\sum_{Y}\left(2 g(1)+\sigma_{Y}-1\right)=h(2 g(1)-1)+\sigma
$$

où la somme porte sur les composantes $Y$ de $\bar{X}(1), \sigma_{Y}$ est le nombre de points supersinguliers sur $Y$ (en fait, tous les $\sigma_{Y}$ sont égaux), et $\sigma=$ $\sum \sigma_{Y}=h(D(p))$. En tenant compte du fait

$$
\left.p_{a} \bar{X}_{0}(\mathfrak{p})\right)=h . g_{0}(\mathfrak{p})=h . g e n r e\left(\bar{M}_{0}(\mathfrak{p}) \times_{A_{H}} H\right),
$$

on obtient

$$
\left.h(D(\mathfrak{p}))=h\left(g_{0}(\mathfrak{p})\right)-2 g(1)+1\right)
$$

Les nombres $g(1)$ et $g_{0}(\mathfrak{p})$ sont calculés dans [7], VI 5.8 et VII 5.13 à l'aide des formes modulaires. Nous renonçons à écrire les formules compliquées ; ils fournissent le résultat

$$
h(D(\mathfrak{p}))=d_{\infty} \cdot P(1)[Q . P(q)+\eta]
$$

avec $\eta=0$ si $d$ ou $d_{\infty}$ est pair, $\eta=\left(q /(q+1)\right.$ ). $P(-1)$ si $d$ et $d_{\infty}$ sont impairs, et $Q$ comme dans l'énoncé du théorème 1 . 


\section{Points elliptiques.}

Un point géométrique de $M(1)$ est dit elliptique s'il correspond à un module de Drinfeld $\Phi$ ayant un groupe d'automorphismes strictement plus grand que $F_{q}^{*}$. Dans ce cas, $\operatorname{Aut}(\Phi)$ est le groupe multiplicatif de l'extension quadratique de $F_{q}$. Cette remarque triviale, combinée avec le théorème 2 (iii) montre déjà (i) du théorème 1 .

Posons $A^{\prime}=A \otimes \mathrm{F}_{q^{2}}$ et $K^{\prime}=\operatorname{Fract}\left(A^{\prime}\right)$, et supposons d'abord que $d_{\infty}$ soit pair . Si le module de $D . \Phi: A \rightarrow L\{\tau\}$ satisferait à $\operatorname{Aut}(\Phi) \cong \mathrm{F}_{q^{*}}^{*}$, l'homomorphisme $\Phi$ aurait une extension à $A^{\prime}$. Mais ceci est absurde parce que, grâce à l'hypothèse, $A^{\prime}$ a un groupe d'unités infini. Donc, si $d_{\infty}$ est pair, il n'existe aucun point elliptique, et nous pouvons supposer dans tout ce qui suit que $d_{\infty}$ soit impair. Alors, la place $\infty$ est inerte dans $K^{\prime}$, et l'on peut regarder les $A$ - modules de Drinfeld de rang 2 avec $\operatorname{Aut}(\Phi) \simeq \mathrm{F}_{q^{2}}^{*}$ comme des $A^{\prime}$-modules de rang 1 et vice-versa. Ceci définit un morphisme

$$
\varepsilon: M_{A^{\prime}}^{1}(1) \rightarrow M^{2}(1)=M(1)
$$

dont l'image consiste en des points elliptiques. Ici, $M_{A^{\prime}}^{1}(1)$ est le schéma de modules des $A^{\prime}$-modules de $D$. de rang 1 "sans niveau". Grâce à [5], Thm. 1 , il est égal au spectre de $A_{H^{\prime}}^{\prime}=A^{\prime}$-entiers dans le corps de classes de Hilbert $H^{\prime}$ de $K^{\prime}$. La conjugaison de $\mathrm{F}_{q^{2}}$ sur $\mathrm{F}_{q}$ induit une involution $\beta$ de $M_{A^{\prime}}^{1}(1)$, vu comme $A$-schéma. Soit $\pi: M_{A^{\prime}}^{1}(1) \rightarrow \operatorname{Spec}\left(A_{\beta}\right)$ le morphisme quotient, où $A_{\beta} \subset A_{H^{\prime}}^{\prime}$ est le sous-anneau des $\beta$-invariants. Alors $\varepsilon$ se factorise en $\varepsilon=\tilde{\varepsilon} \circ \pi$, et $\tilde{\varepsilon}: \operatorname{Spec}\left(A_{\beta}\right) \rightarrow M^{2}(1)$ est une immersion fermée ([7], VII, §2). Vu que $A_{\beta}$ est fini, plat et non ramifié sur $A$, l'image de $\tilde{\varepsilon}$ contient

$$
h^{\prime}=\left[A_{\beta}: A\right]=\left[A_{H^{\prime}}^{\prime}: A^{\prime}\right]=\#\left(\operatorname{Pic} A^{\prime}\right)
$$

points géométriques au-dessus de chaque $\mathfrak{p} \in \operatorname{Spec} A$ (i.e. points elliptiques sur $\left.\bar{F}_{\mathfrak{p}}\right)$. II est facile de voir que $h^{\prime}$ est donné par $d_{\infty} . P(1) \cdot P(-1)$, donc :

(7.1) Proposition : Si $d_{\infty}$ est impair (pair), il existe $d_{\infty} . P(1) . P(-1)$ (resp. nuls) points elliptiques dans $M(1) \times \overline{\mathbf{F}}_{\mathrm{p}}$.

Il reste à voir combien de points elliptiques sont supersinguliers. La réponse est

(7.2) Lemme : Supposons $d_{\infty}$ impair. Un point elliptique de $M(1) \times \overline{\mathbf{F}}_{\mathfrak{p}}$ est supersingulier si et seulement si $d=\operatorname{deg} \mathfrak{p}$ est impair.

DÉmontration : Soient $\mathfrak{q}\left(\right.$ resp. $\left.q_{1}, q_{2}\right)$ les diviseurs dans $A^{\prime}$ de $\mathfrak{p}$, si $d$ est impair (resp. pair). Si Aut $(\Phi) \cong \mathrm{F}_{q^{2}}^{*}, \Phi$ se prolonge en $\Phi^{\prime}: A^{\prime} \rightarrow$ 
$\bar{F}_{q}\{\tau\}$ (resp. en $\Phi^{\prime}: A^{\prime} \rightarrow \bar{F}_{q_{1}}\{\tau\}=\bar{F}_{p}\{\tau\}$ ). En tous cas, $\Phi^{\prime}$ est un $A^{\prime}$ module de $D$. de rang 1 . Si $d$ est impair, ${ }_{p} \Phi={ }_{q} \Phi^{\prime}$ est local, donc $\Phi$ est supersingulier. Si $d$ est pair, on a pour les centralisateurs dans $\bar{F}_{\mathrm{p}}\{\tau\}$ :

$$
\operatorname{Cent}(\Phi(A))=\operatorname{Cent}\left(\Phi^{\prime}\left(A^{\prime}\right)\right)=\Phi^{\prime}\left(A^{\prime}\right) \cong A^{\prime},
$$

ce qui est un $A$-module projectif de rang deux, et $\Phi$ n'est pas supersingulier.

En combinant (7.1), (7.2) avec le théorème 2 et (6.8), on arrive à (ii) du théorème 1.

\section{Exemples.}

(8.1) Considérons le cas de l'exemple 3.2 où $K=\mathrm{F}_{q}(T)$ et $A=\mathrm{F}_{q}[T]$. Alors $P(X)=1$. On a

$$
\begin{aligned}
& h_{1}=\left(q^{d}-1\right) /\left(q^{2}-1\right) \text { et } h_{2}=0, \text { si } d \text { est pair, et } \\
& h_{1}=\left(q^{d}-q\right) /\left(q^{2}-1\right) \text { et } h_{2}=1, \text { si } d \text { est impair. }
\end{aligned}
$$

Le nombre $t(D(\mathfrak{p}))$ de types de $D(\mathfrak{p})$ peut aussi être calculé à l'aide des nombres de classes des extensions quadratiques de $K$ ([8] 4.7).

(8.2) Soit encore $K$ un corps de fonctions rationnelles, mais " $\infty$ " de degré 2 , e.g. le corps de fonctions de la courbe

$$
Y^{2}=a X^{2}+b X+c,
$$

où $p>2, a$ n'est pas carré dans $\mathrm{F}_{q}$, et $b^{2}-4 a c \neq 0$. Alors $h_{1}(D(\mathfrak{p}))=$ $2\left(q^{d}-1\right) /(q-1)$ et $h_{2}(D(\mathfrak{p}))=0$. Si $d=1$, les deux classes de modules supersinguliers sur $\bar{F}_{p}$ sont définies sur l'extension quadratique $F_{p}^{(2)}$ de $F_{p}$ et conjuguées, ce qui résul te de (4.1). Donc par le théorème $2, t(D(p))=1$.

(8.3) Soit $K$ de genre 1 et " $\infty$ " une place de degré 1 . Le polynôme $P(X)$ prend la forme $q X^{2}-t X+1$ avec $|t| \leq 2 q^{1 / 2}$. On a

$$
\begin{aligned}
& \left.h_{1}=P(1) P(q) q^{d}-1\right) /\left(q^{2}-1\right) \text { et } h_{2}=0, \quad \text { si } d \text { est pair, et } \\
& h_{1}=P(1) P(q)\left(q^{d}-1\right) /\left(q^{2}-1\right)-P(1) P(-1) /(q+1) \text { et } h_{2}=(q+1)^{2}-t^{2},
\end{aligned}
$$
si $d$ est impair.

Le nombre $h_{1}$ se simplifie à $(q-t+1)\left(q^{2}-q-t\right)$ pour $d=1$. 


\section{BIBLIOGRAPHIE}

[1] A. BRUMER, Courbes modulaires. Grenoble, (1975).

[2] P. DELIGNE, D. HUSEMÖLlER, Survey of Drinfeld modules, Contemp. Mat. 67 (1987), 25-91.

[3] P. DELIGNE, M. RAPOPORT, Les schémas de modules de courbes elliptiques, Lecture Notes in Mathematics 349. Springer-Verlag, (1973).

[4] M. DEURING, Die Typen der Multiplikatorenringe elliptischer Funktionenkörper, Abh. Hamb. 14 (1941), 197-272.

[5] V.G. DRINFELD, Elliptic modules, Math. USSR-Sbornik 23 (1976), 561-592.

[6] E.-U. GEKELER, Über Drinfeld'sche Modulkurven vom Hecke-Typ, Comp. Math. 57 (1986), 219-236.

[7] E.-U. GEKELER, Drinfeld modular curves, Lecture Notes in Mathematics 1231. Springer-Verlag, (1986).

[8] E.-U. GEKELER, On finite Drinfeld modules, J. Algebra. à paraitre.

[9] M.-F. VIGNÉRAS, Arithmétique des Algèbres de Quaternions, Lecture notes in Mathematics 800. Springer-Verlag, (1980).

Institut des Hautes Etudes Scientifiques

35, route de Chartres

91440 - Bures-sur-Yvette (FRANCE) 